\title{
Maternal repression of the human GRB10 gene in the developing central nervous system; evaluation of the role for GRB10 in Silver-Russell syndrome
}

\author{
Megan P Hitchins ${ }^{*, 1}$, David Monk ${ }^{1,2}$, Gillian M Bell ${ }^{1}$, Zehra Ali $^{1}$, Michael A Preece ${ }^{2}$, \\ Philip Stanier ${ }^{1}$ and Gudrun E Moore ${ }^{1}$
}

${ }^{1}$ Action Research Laboratory for the Molecular Biology of foetal Development, Division of Paediatrics, Obstetrics and Gynaecology, Imperial College School of Medicine, Queen Charlotte's and Chelsea Hospital, Goldhawk Road, London W6 OXG, UK; ${ }^{2}$ Department of Biochemistry, Endocrinology and Metabolism, Institute of Child Health, University College London, 30 Guilford Street, London WC1N 1EH, UK

The GRB10 gene encodes a growth suppressor and maps to human chromosome 7p11.2-p13. Maternal duplication (matdup) of this region has recently been associated with Silver-Russell syndrome (SRS), which is characterised by pre- and postnatal growth restriction, craniofacial dysmorphism and lateral asymmetry. Maternal uniparental disomy for chromosome 7 (mUPD7) occurs in approximately 7\% of SRS patients. Exposure of a recessive allele due to isodisomy has been ruled out in five mUPD7 cases, suggesting genomic imprinting as the basis for disease. Assuming SRS patients with matdup of 7p11.2-p13 and mUPD7 share a common aetiology, this would implicate a maternally expressed gene from this interval, which is involved in growth inhibition. Murine Grb10 was identified as a maternally expressed gene by subtractive hybridisation using normal and androgenetic mouse embryos. Grb10 maps to the homologous region of proximal mouse chromosome 11, for which mUPD incurs reduced birthweight. A role for GRB10 in SRS was evaluated by determining its imprinting status in multiple human foetal tissues using expressed polymorphisms, and by screening the coding region for mutations in 18 classic non-mUPD7 SRS patients. Maternal repression of GRB10 was observed specifically in the developing central nervous system including brain and spinal cord, with biallelic expression in peripheral tissues. This is in contrast to mouse Grb10, and represents the first example of opposite imprinting in human and mouse homologues. While a role for GRB10 in mUPD7 SRS cases can not be ruled out on the basis of imprinting status, no mutations were identified in the patients screened. European Journal of Human Genetics (2001) 9, 82-90.

Keywords: GRB10; SRS; tissue-specific; genomic imprinting

Introduction

Silver-Russell syndrome (SRS) [MIM 180860] is primarily a growth disorder, characterised by pre- and postnatal growth

*Correspondence: Dr Megan P Hitchins, Action Research Laboratory for the Molecular Biology of foetal Development, Division of Paediatrics, Obstetrics and Gynaecology, Imperial College School of Medicine, Queen Charlotte's and Chelsea Hospital, Goldhawk Road, London W6 OXG, UK. Tel: +44 208383 3533; Fax: +44 208383 1838; E-mail: mhitchin@hgmp.mrc.ac.uk

Received 15 May 2000; revised 4 September 2000; accepted 22 September 2000 restriction, relative sparing of cranial growth, triangular facies, lateral asymmetry, fifth finger clinodactyly and mild cognitive deficit in some cases. ${ }^{1-3}$ The genetic aetiology of SRS is heterogeneous, but maternal uniparental disomy for chromosome 7 (mUPD7) accounts for approximately 7\% of patients. $^{4-6}$ Lack of consistent isodisomy for 40 loci distributed over the full-length chromosome 7 has been demonstrated in a group of five mUPD7 cases, indicating that SRS is due to an imprinting effect, as opposed to unmasking of a recessive allele. ${ }^{7}$

Recently, two patients with classical features of SRS have been described, each with a maternal duplication of $7 \mathrm{p} 11.2$ - 
p13. ${ }^{8,9}$ Assuming the duplicated regions in these two patients are functionally active, this suggests that the aetiological basis for SRS in mUPD7 cases may be over-expression of a maternally expressed gene involved in growth suppression, rather than lack of a paternally-derived growth promoter. The duplications, which contain the insulin-like growth factor binding protein 1 and 3 genes (IGFBP1 and 3) and GRB10, define a candidate region for SRS on the short arm of chromosome 7 . The IGFBP 1 and 3 genes are not imprinted in adult and foetal tissues ${ }^{10,11}$ and no mutations were detected in either gene in a cohort of non-mUPD7 SRS patients, ${ }^{12}$ making these unlikely candidates for this disorder. GRB10 has previously been proposed as a candidate for the SRS phenotype on the basis of its protein function as a growth suppressor, its chromosomal location and imprinting status in the mouse. ${ }^{13}$

GRB10 contains a central pleckstrin homology $(\mathrm{PH})$ domain and a carboxy-terminal Src homology 2 (SH2) domain. The human and mouse orthologues are highly conserved, with 88 and 99\% amino acid similarity in the $\mathrm{PH}$ and $\mathrm{SH} 2$ domains respectively, and 70-99\% similarity in the remaining segments of the two proteins. ${ }^{14-16}$ Four GRB10 isoforms have been identified in humans, which differ at the amino-terminus and the $\mathrm{PH}$ domain due to alternative splicing events (Figure 1); GRB10 $\alpha$ (previously referred to as GRB-IR), ${ }^{14}$ GRB10 $\beta$ (or GRB-IRSV1), which is the most widely and abundantly expressed isoform, ${ }^{15,16}$ GRB10 $\gamma,{ }^{17}$ and GRB108, which has an extended 3' untranslated region.

GRB10 has been shown to interact with several tyrosine kinase growth factor receptors including the insulin ${ }^{14-16}$ and insulin-like growth factor-I (IGF-I) ${ }^{15,18}$ receptors, and the platelet-derived growth factor (PDGF) receptor, ${ }^{16}$ and so may serve a broad role in growth suppression through various receptor pathways. Over-expression of the murine Grb10 $\alpha$ has been shown to inhibit the tyrosine phosphorylation of two endogenous insulin receptor substrates upon insulin stimulation. ${ }^{14}$ An excess of this protein was also shown to cause suppression of IGF-I mediated cell proliferation in mouse fibroblasts. ${ }^{19}$ The SH2 domain of human GRB10 has been shown to inhibit both insulin and IGF-I stimulated mitogenesis in fibroblast cell lines. ${ }^{15,17}$ GRB10 also interacts with the non-tyrosine kinase growth hormone $(\mathrm{GH})$ receptor, causing down regulation of the $\mathrm{GH}$ signalling pathway. ${ }^{20}$

The region of human chromosome 7p11.2-p13 containing $G R B 10,{ }^{21}$ is homologous to an imprinted region on mouse proximal chromosome 11 , and mice with a maternal duplication/paternal deficiency of this region are growth restricted. $^{22}$ Mouse Grb10 was identified in a systematic screen for maternally expressed genes by subtractive hybridisation, and is a candidate for this growth phenotype. ${ }^{13}$ Asynchronous replication has been demonstrated at the human GRB10 locus in normal lymphocytes and lymphoblasts, giving a preliminary indication that the human homologue is also imprinted. ${ }^{9}$

To determine if GRB10 contributes to the SRS phenotype, firstly the imprinting status of the gene during human foetal development was investigated by direct analysis of allelic transcription using intragenic polymorphisms. We demonstrate repression of expression from the maternal allele for human GRB10 specifically in the foetal central nervous system (CNS), accompanied by biallelic expression in all other major foetal tissues. This is in contrast to the maternally expressed mode of imprinting of the mouse homologue. ${ }^{13}$ Secondly, a group

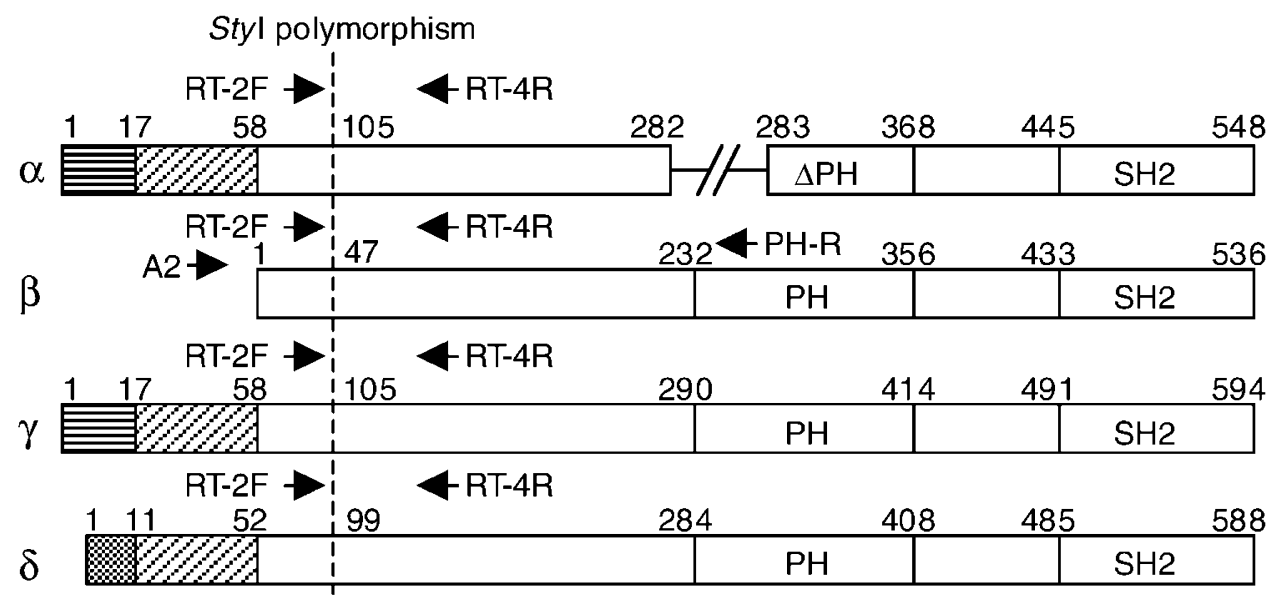

Figure 1 GRB10 protein isoforms. Schematic of the four known GRB10 isoforms aligned according to regions of similarity. GRB10 $\alpha$ (GRB-IR) has an incomplete PH domain with a 46-amino acid deletion due to skipping of exon 7A. GRB10 $\beta$ has a short amino terminus and complete PH domain. GRB10 $\gamma$ has the same amino terminus as GRB10 $\alpha$, but with a full-length PH domain. The GRB10 $\delta$ CDNA encodes a unique amino terminus and full PH domain. Regions common to all isoforms are represented by white boxes. Patterned boxes denote the different amino termini. Amino acid residues are numbered. A polymorphic Styl site in exon 3, used for imprinting analysis, is indicated by a vertical dashed line. The positions of RT - PCR primers with respect to the protein isoforms are indicated by arrows. 
of 18 classical SRS patients in whom mUPD7 and cytogenetic abnormalities had been ruled out, were analysed for mutations of the coding region of GRB10. No mutations were detected in any of the patients, but two novel polymorphisms were identified. Thus, while an imprinting effect of GRB1O on SRS patients can not be ruled out, the lack of any mutations in these patients suggests that this gene is neither likely to contribute to SRS in a significant number of patients, nor be responsible for the full disease spectrum.

\section{Materials and methods}

Tissue samples for GRB10 imprinting analysis

Foetal tissues were obtained from terminated pregnancies at Queen Charlotte's and Chelsea Hospital (QCCH). Samples were washed in sterile PBS prior to freezing in liquid nitrogen. Corresponding maternal peripheral blood was also acquired at QCCH. Local ethics approval for obtaining foetal and maternal samples was granted by the Research Ethics Committee of the Royal Postgraduate Medical School (96/4955), and informed consent was obtained for each sample pair. Normal fibroblasts were obtained from anonymous volunteers by 3-mm punch skin biopsy.

\section{SRS patient and normal control samples}

Eighteen classical SRS patients (demonstrating at least three of the following characteristics; low birthweight (at least 2SD below the mean), short stature (at least -2SD), lateral asymmetry and characteristic craniofacial dysmorphism) were screened for mutations of GRB10. These patients were a subset of 53 previously described. ${ }^{7}$ Major structural abnormalities including duplications, inversions and translocations had previously been excluded by cytogenetic analysis. Trisomy mosaicism and mUPD for chromosome 7 had also been ruled out by microsatellite repeat analysis of the nuclear pedigrees. Peripheral blood samples for DNA analysis were obtained with the patients' informed consent. This study was approved by the Joint Research Ethics Committee of the Great Ormond Street Hospital and the Institute of Child Health (1278). Genomic DNA from peripheral blood of 10 anonymous normal individuals served as controls.

\section{PCR amplification of genomic DNA}

Genomic DNA was extracted from placenta and peripheral blood using standard phenol-chloroform separation. ${ }^{23}$ For PCR analysis, $200 \mathrm{ng}$ genomic DNA was amplified in $50-\mu \mathrm{l}$ reactions containing $1 \times \mathrm{NH}_{4}$ reaction buffer, $50 \mathrm{ng}$ each oligonucleotide primer, $0.2 \mathrm{~mm}$ each dNTP, $1.5 \mathrm{~mm} \mathrm{MgCl}_{2}$ and $2 \mathrm{U}$ DNA Taq DNA Polymerase (Bioline). PCR was performed for 35 cycles of denaturation at $94^{\circ} \mathrm{C}$ for $60 \mathrm{~s}$, followed by $45 \mathrm{~s}$ annealing (temperature as stipulated for each primer pair), and extension at $72^{\circ} \mathrm{C}$ for $60 \mathrm{~s}$.

\section{Analysis of expression by reverse-transcription (RT) - PCR}

Total RNA was isolated from foetal tissues and normal adult fibroblasts using the guanidium isothiocyanate extraction technique. ${ }^{24}$ For RT-PCR, $2 \mu \mathrm{g}$ denatured total RNA from each sample was used to synthesise cDNA in $20-\mu 1$ reactions with $1 \times$ RT buffer, $40 \mathrm{U}$ M-MLV reverse transcriptase (Promega), 1 unit RNase inhibitor (Promega), $1 \mathrm{~mm}$ each $\mathrm{dNTP}$, and $0.2 \mu \mathrm{g}$ primer at $37^{\circ} \mathrm{C}$. Poly-dT primed cDNA was used for study of the GRB1O $3^{\prime}$ UTR, and random hexamers were used to produce cDNA for analysis of the GRB10 coding region. A duplicate set of samples was processed, with RT omitted to detect any genomic DNA contamination of the RNA. One tenth of the resulting cDNA and RT samples were subjected to PCR amplification, as above. The number of cycles varied such that PCR reactions were terminated in the log-linear phase (to minimise any allelic bias in amplification). RT-PCR primers were designed to amplify between exons, across at least one intron, to detect amplification of genomic contamination or antisense RNA on the basis of product size.

\section{Sequencing}

PCR and RT - PCR samples were purified for sequencing using MicroSpin S-300 HR columns (Pharmacia). Sequencing was performed using the BigDye Terminator ready reaction kit on an automated ABI PRISM 377 DNA sequencer (PE Biosystems).

\section{Analysis of GRB10 imprinting}

A StyI polymorphism within GRB10 exon 3 was used to study the allelic origin of expression of GRB10, and specifically the GRB10 $\beta$ isoform (GenBank: U69276). To identify informative foetal-maternal pairs, genomic DNA extracted from placenta and maternal blood was PCR amplified using intronic primers $3 \mathrm{~F}$ and $3 \mathrm{R}$ (Table 1 ) and analysed by restriction digestion with StyI. Digestion reactions were performed using $5 \mu \mathrm{l}$ PCR product with $1 \times$ Buffer F and $10 \mathrm{U}$ StyI (Promega) in $20-\mu \mathrm{l}$ volumes at $37^{\circ} \mathrm{C}$ for $3 \mathrm{~h}$. For RT - PCR analysis of all GRB10 isoforms concomitantly, a forward primer from within exon 2 (RT-2F: AGTCTGACCGACTTGCGAAT) and reverse primer from exon 4 (RT-4R: CCACAGAGTTCAGGAAAAGG), were used to amplify cDNAs from heterozygous foetal tissues with annealing at $60^{\circ} \mathrm{C}$. To study the expression of the $G R B 10 \beta$ isoform individually, GRB10 $\beta$-specific forward primer A2 (AGACCTAAGCCTGTTTGCTC) ${ }^{16}$ and reverse primer PH-R (CAGGACAACTACTGGAGTTC) were used with annealing at $56^{\circ} \mathrm{C}$. Between 6 and $15 \mu \mathrm{l}$ RT - PCR product was digested with StyI to determine imprinting status.

Imprinting of the GRB10 (GenBank: D86962) mRNA was analysed using a novel $\mathrm{G} \rightarrow \mathrm{A}$ polymorphism present in the extended 3' UTR of GRB10 $\delta$. Genomic DNA was amplified using primers from within the extended exon 15 sequence, which flanked the polymorphism (15-3'F: GTGATAAAGACAAGGAAACG and 15-3'R: AAACAACTAGAGTGTGGTCT), 
Table 1 Primers used for PCR amplification and sequencing of GRB10 from genomic DNA

\begin{tabular}{|c|c|c|c|c|}
\hline Exon number & Primer sequences $\left(5^{\prime}\right.$ to $\left.3^{\prime}\right)$. Forward $(F)$, Reverse $(R)$ & $\mathrm{Tm}^{\circ} \mathrm{C}$ & Product size (bp) & GenBank number \\
\hline 1 & $\begin{array}{l}\text { 1F: GGCTTGGCTTCTCACAGTCTG } \\
\text { 1R: ATCTATGGCTGGTGGCGACAT }\end{array}$ & 58 & 213 & AF073363 \\
\hline 2 & 2F: TTCTGCTGCGGTCCTGTTTTT ${ }^{a}$ & 55 & 159 & AF073364 \\
\hline 3 & $\begin{array}{l}\text { 3F: CCTGATCACCAAGATGTACA } \\
\text { 3R: GAGGCCTGGACCTACCTG }\end{array}$ & 58 & 321 & AF073365 \\
\hline 4 & $\begin{array}{l}\text { 4F: GTGTAAGGCTGGGTCAT } \\
\text { 4R: ACTTCCCGCCCTTCTTCC }\end{array}$ & 58 & 298 & AF073366 \\
\hline 6 & $\begin{array}{l}\text { 6F: TACCATGAATTTCCCACCTGT } \\
\text { 6R: TGATACTATGAAAACCCAAGT }\end{array}$ & 53 & 248 & AF073368 \\
\hline 7 & $\begin{array}{l}\text { 7F: CTTTGAAGCTAACCTTTTACG } \\
\text { 7R: CAAGAGGATTTCTATTCTGAA }\end{array}$ & 51 & 172 & AF073369 \\
\hline $7 \mathrm{~A}$ & $\begin{array}{l}\text { 7AF: GCTGATAACATGTCTGCTTA } \\
\text { 7AR: ATTAGGGCTGGTGGTGGTAGC }\end{array}$ & 57 & 242 & AF073370 \\
\hline 11 & $\begin{array}{l}\text { 11F: CTGCGGCCTTTCCTTTTC } \\
\text { 11R: GGCTACCACCTTGAGGGT }\end{array}$ & 61 & 188 & AF073374 \\
\hline 12 & $\begin{array}{l}\text { 12F: GTCTGCTGTCCTCGGTGCTAA } \\
\text { 12R: GGGGTGCTGTTTGATTTTCTT }\end{array}$ & 58 & 164 & AF073375 \\
\hline 13 & $\begin{array}{l}\text { 13F1: CTCTGAGTTGATCAGAGTTG } \\
\text { 13R1: TTAGCAGATGACAGCTCTTA }\end{array}$ & 54 & 216 & AF073376 \\
\hline 14 & $\begin{array}{l}\text { 14F1: CAGAATTACCGTCTTGGTGC } \\
\text { 14R1: CTGTCTGATACTTACCGGTC }\end{array}$ & 55 & 416 & AF073377 \\
\hline 15 & $\begin{array}{l}\text { 15F1: СтGCCTCTAACCTGGCAAGTG } \\
\text { 15R1: СтGGATCTTCCATGCCCTCC }\end{array}$ & 60 & 428 & AF073378 \\
\hline
\end{tabular}

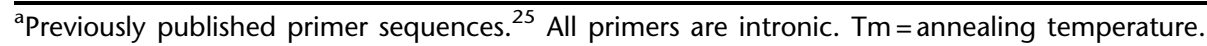

at an annealing temperature of $52^{\circ} \mathrm{C}$. The PCR products were sequenced across the polymorphic site with $15-3^{\prime}$ R. For RT PCR analysis, a 1.932-kb cDNA product was amplified using a forward primer from coding exon 14 (RT-14F: CAGAGTAATCCAAAGGCATT) and reverse primer $15-3^{\prime} \mathrm{R}$, with annealing at $53^{\circ} \mathrm{C}$ and $1.75 \mathrm{~mm} \mathrm{MgCl}_{2}$. RT-PCR products were sequenced with primers $15-3^{\prime} \mathrm{F}$ and $15-3^{\prime} \mathrm{R}$ to determine the allelic origin of expression.

\section{Screen for GRB10 mutations in SRS patients}

GRB10 exons $1-15^{25}$ were PCR amplified from genomic DNA of 18 SRS patients and 10 anonymous normal controls using primers listed in Table 1 . The PCR products were directly sequenced. SRS patient sequences were analysed for mutations and polymorphisms using the Sequence Navigator program, and through direct comparison to the published GRB10 exon sequences (Table 1) by BLAST analysis.

\section{Results}

Tissue-specific imprinting of GRB10

The imprinting status of GRB10 was studied during the first and second trimesters of foetal development and in adult fibroblasts by exploiting expressed sequence polymorphisms to distinguish the parental alleles. The allelic origin of expression was traced in the cDNA of heterozygous foetal tissue samples for which the corresponding maternal allele was known.

Initially, the imprinting status of the undifferentiated GRB10 transcripts was studied by RT-PCR using primers capable of co-amplifying each splice variant across a previously described StyI polymorphism within exon $3,^{25}$ that is present in all four known isoforms (Figure 1). Imprinting of GRB10 was thus studied by restriction digestion of the amplified cDNAs. In total, 68 foetuses were screened for heterozygosity, as detected by presence of both the $319 \mathrm{bp}$ undigested StyI-PCR product and the StyI+ digested fragments of 257 and $62 \mathrm{bp}$ (Figure 2A). GRB10 imprinting was studied in 19 heterozygous foetuses, which ranged from 7-15 weeks gestation, for which a variety of tissues were available. Nine of the sample sets were informative with respect to parental origin of the alleles, with four maternal samples homozygous for the StyI - allele and five homozygous for the StyI+ allele.

The GRB10 cDNAs from heterozygous tissue samples were RT - PCR amplified using primers flanking the polymorphism and introns 2 and 3, and digested with StyI (Figure 2B). In the majority of foetal tissues, including vertebral column, eye, 

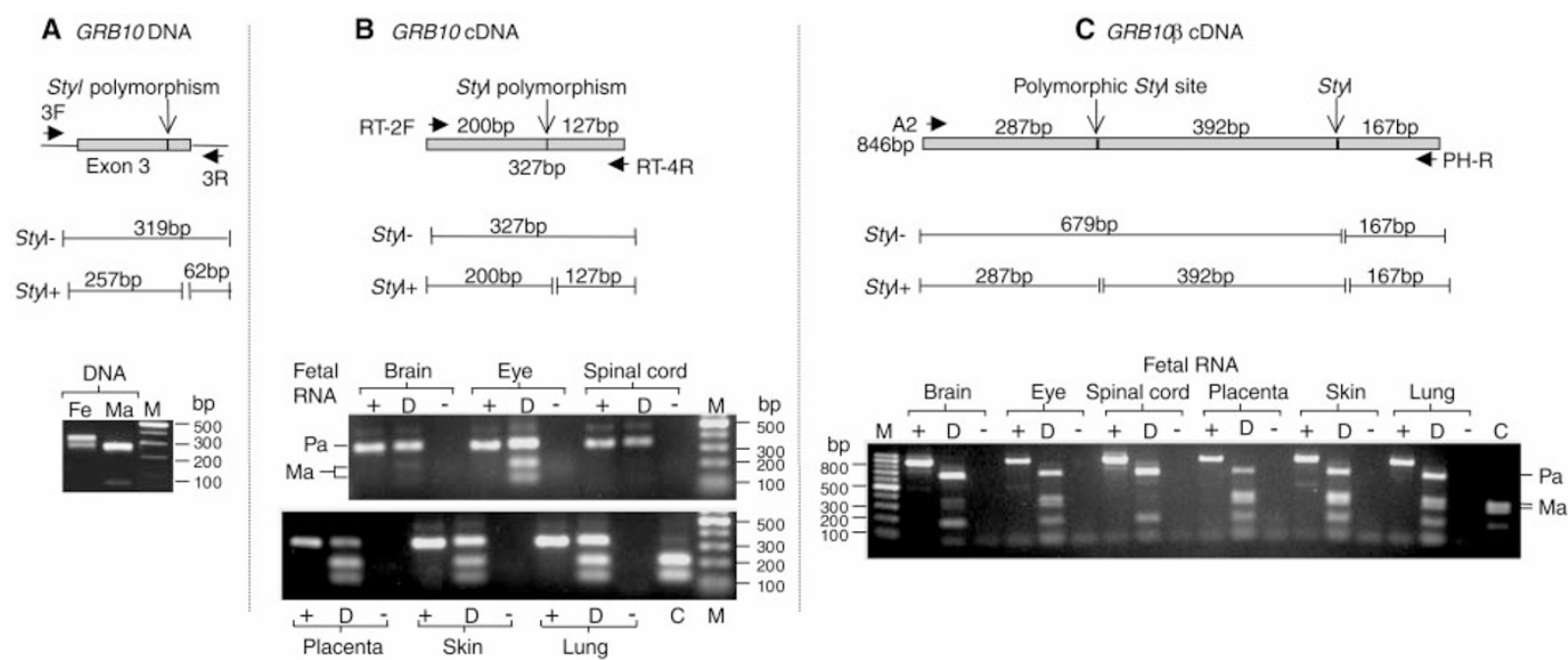

Figure 2 Imprinting analysis of $G R B 10$ and the $G R B 10 \beta$ isoform in human foetal tissues. (A) Informative foetal-maternal pair for the GRB10 exon 3 Styl polymorphism. Top: schematic of exon 3 amplification product from genomic DNA using intronic primers 3F and 3R, and the position of the Styl polymorphism. Fragment sizes of the Styl+ and Styl- alleles are shown beneath. Bottom: Electrophoresed Styl digested PCR products of heterozygous 11-week foetal DNA (Fe) and the corresponding maternal DNA (Ma), which is homozygous for the Styl+ allele. (B) RT-PCR imprinting assay of undifferentiated GRB10 cDNA in tissues from foetus in A. Top: schematic of undifferentiated GRB10 CDNA amplified with primers RT-2F and RT-4R across the Styl polymorphism, and its restriction fragment sizes for the Styl+ and Styl-alleles. Bottom: Gel containing Styl digested RT-PCR products of GRB10 CDNA. The paternally derived (Pa) Styltranscripts and maternal Styl+ bands are indicated. The paternal allele is predominant in brain and the only allele visualised in spinal cord. Both parental alleles were detected in the RNA of all other tissues. A homozygous Styl+ control to demonstrate complete Styl digestion (C) was included. (C) Imprinting assay for GRB1O $\beta$ isoform in same foetal samples. Top: Styl restriction map of the GRB10 $\beta$ RT - PCR product, showing the positions of the polymorphic and non-polymorphic Styl sites, and the restriction fragments generated for the Styl+ and Styl- alleles. Bottom: electrophoresed Styl digested GRB10 R RT-PCR products. The 679 bp Styl - paternal allele only is detected in spinal cord and is predominant in brain. The same Styl digestion control, (C) was used. (D) amplification products digested with Styl. (+) reactions containing RT. (-) RT negative reactions. (M) 100 bp size marker.

tongue, heart, lung, kidney, stomach, intestine, pancreas, adrenals, skin, limb, placenta, umbilical cord and yolk sac, expression was detected from both parental alleles (Figure 2B and Table 2). GRB10 is widely expressed, but is most abundant in insulin responsive tissues including the pancreas, skeletal muscle and adipocytes. ${ }^{14,16}$ Biallelic expression was clearly observed in the pancreas. Although GRB10 expression in the limb and skin was also biallelic, these tissues are relatively heterogeneous, so that skeletal muscle and adipocytes were not examined separately. Both parental alleles were similarly observed in adult fibroblasts (Table 2). In eight foetal brain and three spinal cord informative samples, a very different pattern of GRB10 expression was observed, with distinct repression of the maternal allele (Figure 2B, Table 2). In spinal cord of 8-12 weeks post-conception, expression was detected exclusively from the paternal allele. In foetal brain, expression occurred predominantly or monoallelically from the paternal allele. Since the RT-PCR assay was not quantitative, the levels of maternally derived transcripts could not be accurately assessed. This imprinted pattern of expression in the CNS was observed irrespective of whether the expressed allele was the StyI+ or StyI - genotype.
Analysis of imprinting in individual GRB10 isoforms Isoform-specific imprinting, through the utilisation of different promoters, has been demonstrated in the $I G F 2^{26}$ and $M E S T^{27}$ genes. In the case of the paternally expressed MEST gene, biallelic expression was observed specifically in lymphocytes. $^{27}$ Recently the existence of a second nonimprinted isoform in lymphocytes was reported, which had previously masked the imprinted expression of the original isoform. ${ }^{28}$ The possibility of isoform-restricted imprinting of GRB10 was investigated using isoform-specific RT-PCR.

To examine the allelic expression of the ubiquitous GRB10 $\beta$ isoform (GenBank: U69276) individually, RT-PCR was performed using forward primer A2 from the 5' UTR unique to $G R B 10 \beta^{16}$ and reverse primer from exon $7 \mathrm{~A}$ (Figure 1 ). The amplified cDNA included the exon 3 polymorphic StyI site, but also incorporated a second non-polymorphic StyI site (Figure 2C). Imprinting analysis of GRB10 $\beta$ was performed using the same foetal-maternal sample pairs and adult fibroblasts used for analysis of the undifferentiated GRB10 transcripts. GRB10 $\beta$ was also expressed exclusively from the paternal allele in spinal cord and predominantly or monoallelically from the paternal allele in the brain (Figure 2C, Table 2). Samples demonstrating preferential expression were 
Table 2 Allelic origin of expression of GRB10, and individual GRB10 isoforms $\beta$ and $\delta$, in foetal tissues and adult cells

\begin{tabular}{llll}
\hline Tissue & $\begin{array}{l}\text { Allelic expression of GRB10 } \\
\text { using Styl polymorphism }\end{array}$ & $\begin{array}{l}\text { Allelic expression of GRB10 } \beta \\
\text { using Styl polymorphism }\end{array}$ & $\begin{array}{l}\text { Allelic expression of GRB10 } \delta \\
\text { studied with } 3^{\prime} \text { UTR polymorphism }\end{array}$ \\
\hline Brain & $6 / 8$ preferential paternal & $6 / 8$ preferential paternal & $4 / 5$ preferential paternal \\
& $2 / 8$ paternal only & $2 / 8$ paternal only & $1 / 5$ paternal only \\
Spinal cord & 1 uninformative monoallelic & 1 uninformative monoallelic & 1 uninformative monoallelic \\
Vertebral column & $3 / 3$ paternal only & $3 / 3$ paternal only & - \\
Eye & $3 / 3$ biallelic & $3 / 3$ biallelic & $8 / 8$ biallelic \\
Tongue & $6 / 6$ biallelic & $6 / 6$ biallelic & - \\
Heart & $2 / 2$ biallelic & $2 / 2$ biallelic & $6 / 6$ biallelic \\
Lung & $5 / 5$ biallelic & $5 / 5$ biallelic & $4 / 5$ biallelic \\
Liver & $5 / 5$ biallelic & $5 / 5$ biallelic & $3 / 3$ biallelic \\
Kidney & - & - & $2 / 2$ biallelic \\
Stomach & $3 / 3$ biallelic & $3 / 3$ biallelic & $2 / 2$ biallelic \\
Intestine & $2 / 2$ biallelic & $2 / 2$ biallelic & $6 / 6$ biallelic \\
Pancreas & $6 / 6$ biallelic & $6 / 6$ biallelic & - \\
Adrenals & $5 / 5$ biallelic & $5 / 5$ biallelic & - \\
Skin & $5 / 5$ biallelic & $5 / 5$ biallelic & $7 / 7$ biallelic \\
Limb & $5 / 5$ biallelic & $5 / 5$ biallelic & $4 / 4$ biallelic \\
Placenta & $2 / 2$ biallelic & $2 / 2$ biallelic & $7 / 7$ biallelic \\
Umbilicus & $9 / 9$ biallelic & $9 / 9$ biallelic & $1 / 1$ biallelic \\
Yolk sac & $3 / 3$ biallelic & $3 / 3$ biallelic & - \\
Adult fibroblasts & $4 / 4$ biallelic & $4 / 4$ biallelic & $1 / 1$ biallelic \\
\hline Niclic & $2 / 2$ biallelic & $2 / 2$ biallelic &
\end{tabular}

Note: all tissues are foetal, except where stated.

concordant for the $\beta$ splice form and the mixed GRB10 isoforms. Biallelic expression was observed in all other foetal tissues tested (Figure 2D) and adult fibroblasts (Table 2).

Imprinting of the GRB10 $\delta$ isoform (GenBank: D86962) was analysed using a novel $\mathrm{G} \rightarrow \mathrm{A}$ polymorphism at nucleotide 4001 of the GRB10 $\delta$ cDNA (Figure 3 ). This occurs in the elongated 3' UTR of the $\delta$ cDNA, which is contiguous with the previously reported exon 15 sequence in genomic DNA (data not shown). Although this polymorphism occurs beyond the extent of the other reported GRB10 isoforms through alternative polyadenylation signalling, it may not be unique

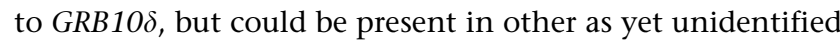
splice variants. The frequency of the 'A' allele was $21 \%$ in 52 foetuses screened for informative heterozygotes. In total, tissue samples from 13 heterozygous fetuses were analysed

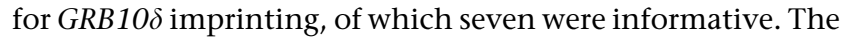
maternal DNA in four sample pairs was homozygous for the ' $G$ ' allele and in three cases, homozygous for the 'A' allele. Three of the foetuses were also heterozygous for the exon 3 StyI polymorphism and had previously been used to analyse the undifferentiated GRB10 and GRB1O $\beta$ imprinting status. The gestational ages of the foetuses analysed were between 7 and 18 weeks. RT - PCR of GRB10 $\delta$ was performed from exon 14 within the coding region, across the G/A polymorphism, to the extended 3' UTR. Allelic origin of expression was examined by automated sequencing of the RT - PCR products across the polymorphic site. As was the case for GRB10 and $G R B 10 \beta$, the GRB10 $\delta$ transcript was expressed predominantly or specifically from the paternal allele in 7-18-week foetal brain (Figure 3, Table 2). This pattern of expression was consistently observed in the sequences in both the forward and reverse-complement directions, whether the paternal allele was a ' $G$ ' or an 'A'. All other foetal tissues tested demonstrated biallelic expression (Table 2), with equal heights of both the $G$ and $A$ peaks in the sequence electropherograms (Figure 3). Biallelic expression was also detected in the adult fibroblasts from a single individual. Thus the imprinting profiles observed for the GRB10 $\beta$ and $G R B 10 \delta$ isoforms were similar to those detected in the undifferentiated GRB10 cDNAs (Table 2) suggesting that imprinting of GRB10 is co-ordinately controlled in neural tissues.

\section{Mutation screening of GRB10 in SRS cases with unknown genetic aetiology}

Eighteen classic SRS patients, in whom cytogenetically distinguishable abnormalities and mUPD7 had been excluded, were analysed for disease-causing mutations of all 16 exons $^{25}$ of the GRB10 gene by sequencing. No mutations were detected in any of the 18 SRS patients screened, when compared to the previously published GRB10 exon sequences (Table 1). However, two novel polymorphisms were identified in GRB10 which were also identified amongst 10 normal controls (Table 3 ). These polymorphisms were predicted to have no effect on splicing events and did not change any amino acid.

\section{Discussion}

We have consistently demonstrated paternal expression of GRB10 in the developing CNS, accompanied by biallelic transcription in a wide range of other organs and peripheral tissues. In the foetal spinal cord, expression was clearly monoallelic from the paternal allele, but in the majority of 
Fetal DNA

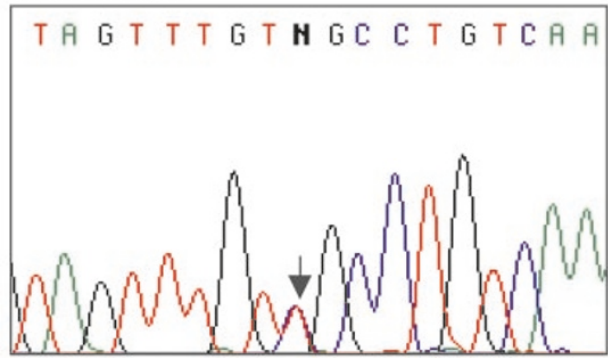

Fetal heart cDNA

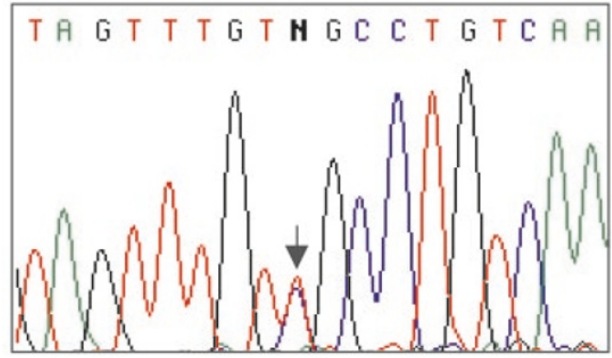

Maternal DNA

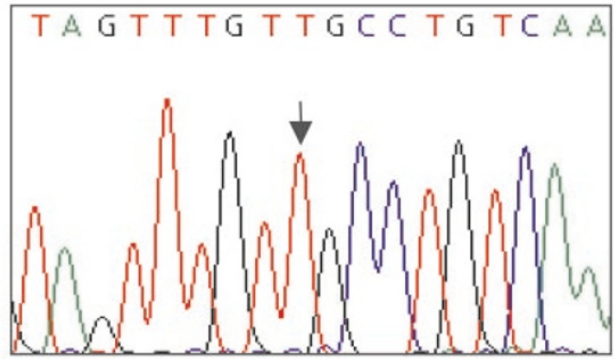

Fetal brain cDNA

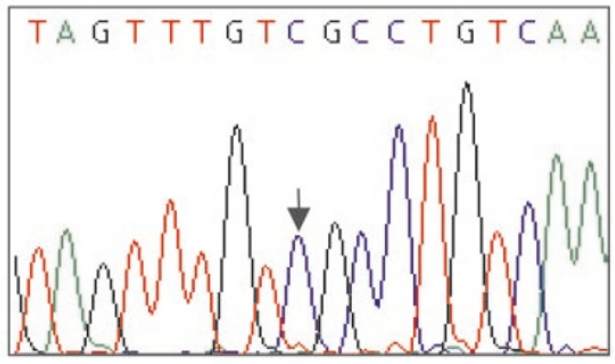

Figure 3 Imprinting analysis of the GRB10 $\delta$ isoform. Sequence electropherograms are shown across nucleotides $4392-4409$ of $G R B 10 \delta$ (GenBank: D86962) for PCR and RT-PCR products from a single informative foetal-maternal pair. Sequences were acquired with primer $15-3^{\prime} \mathrm{R}$ and are in the reverse-complement direction. Arrows indicate the position of the newly identified $\mathrm{G} \rightarrow \mathrm{A}$ polymorphism at nucleotide 4001. The maternal allele of the heterozygous foetus is a ' $T$ '. The brain RNA was monoallelic for the paternally derived ' $\mathrm{C}$ ' allele. Both parental alleles were detected equally in the heart CDNA, illustrating the biallelic expression pattern observed in all other tissues tested.

Table 3 Polymorphisms identified in GRB10 in SRS and normal individuals

\begin{tabular}{lllll}
\hline Site & $\begin{array}{l}\text { Nucleotide } \\
(\mathrm{n}) \text { change }\end{array}$ & $\begin{array}{l}\text { Amino acid } \\
\text { change }\end{array}$ & $\begin{array}{l}\text { Enzyme } \\
\text { change }\end{array}$ & $\begin{array}{l}\text { Number of } \\
\text { alleles tested } \\
\text { frequency }\end{array}$ \\
\hline Exon $3{ }^{\mathrm{a}}$ & $428 \mathrm{G} \rightarrow \mathrm{A}$ & $\mathrm{P} 47 \mathrm{P}$ & Styl & $62 \%$ \\
Intron 4 & $\mathrm{IVS}+35 \mathrm{G} \rightarrow \mathrm{A}$ & - & - & $23 \%$ \\
Exon 11 & $1460 \mathrm{G} \rightarrow \mathrm{A}$ & $\mathrm{A} 391 \mathrm{~A}$ & - & $19 \%$ \\
\hline
\end{tabular}

aPreviously published. ${ }^{25}$ Exonic polymorphism sites are given with respect to the GRB10 $\beta$ cDNA or protein isoform (GenBank U69276).

foetal brain samples analysed, there was incomplete repression of the maternal allele. Although the RT - PCR assays were not strand-specific, such that amplification of a putative antisense transcript was not precluded, the amplification products crossed at least one intron and were of the correct cDNA size. Antisense RNAs have now been described for several imprinted genes, ${ }^{29-31}$ but in each case these have contained intronic sequences not detected in our RT-PCR assays, making it unlikely that we were measuring antisense expression.

The pattern of tissue-specific imprinting of GRB10 strongly resembles that of the Angelman syndrome gene $U B E 3 A$, except the latter is expressed predominantly from the maternal chromosome in brain. ${ }^{32,33}$ In situ hybridisation studies in mice with reciprocal disomies for the region containing Ube3a showed that monoallelic maternal expression occurred in specific regions of the brain, including hippocampus and Purkinje cells, whereas other regions demonstrated preferential maternal or biallelic Ube3a expression. ${ }^{34}$ A similar situation may occur for GRB10 to give an overall pattern of predominant paternal expression in brain. Incomplete silencing of the maternal GRB10 allele could also account for the trace of maternal transcripts detected. The p5 $7^{\mathrm{KIP} 2}$ gene demonstrates preferential expression from the maternal allele in several foetal tissues. ${ }^{35}$ Alternatively, specific GRB10 isoforms may be biallelically expressed in brain. However, two of the four known isoforms, GRB10 $\beta$ and $\delta$, similarly demonstrated monoallelic or predominant expression from the paternal allele in foetal brain. This suggests that regulation of GRB10 imprinting in the CNS is not isoform-specific.

The imprinting status of the human GRB10 gene dramatically contrasts with the maternal-specific expression of mouse Grb10. ${ }^{13}$ The Igf $2 r$ gene is expressed monoallelically 
from the maternal allele in the mouse, but in a polymorphic manner in humans, indicating a partial loss of imprinting during evolution. ${ }^{36}$ However, GRB10 is the first gene to be identified for which contrasting imprinting between the two species has been described. This confounds the arguments surrounding the evolutionary significance of genomic imprinting.

Paternal-specific expression in the foetal brain has also recently been reported by Blagitko et al ${ }^{37}$ corroborating our data: In their study, similar RT-PCR analyses of expressed polymorphisms in foetal tissues for which parental DNA was available were performed. However, a conflicting report published very recently by Yoshihashi et al ${ }^{38}$ states that monoallelic expression of GRB10 is likely to be of maternal origin in the brain. In their study, GRB10 expression was found to be monoallelic in brain, but no corresponding parental DNA samples were available to determine the allelic origin of transcription in this tissue. Instead, in vitro RT - PCR assays were performed in somatic cell hybrids derived from human lymphoblasts and Chinese Hamster ovary cells, containing either a maternal or a paternal human chromosome 7, to determine which parental allele was active. GRB10 expression was detected solely in the cell line with the maternal human 7 , so paternal repression in brain was inferred. ${ }^{38}$ While GRB10 may feasibly be maternally expressed in the somatic cell hybrid system, we and Blagitko et $a l^{37}$ have clearly demonstrated that expression is paternally derived in brain through direct analysis of allelic transcription.

The role GRB1O might play in the aetiology of SRS involving chromosome 7 is difficult to predict in view of the imprinting profile of this gene. Absence of an active paternal allele in the developing CNS may contribute to certain aspects of the clinical phenotype, specifically in mUPD7 patients. However, this would not be the case for patients with matdup 7p11.2-p13, as a normal paternal homologue is present. A novel maternally expressed splice variant, $G R B 10 \gamma-1$, has very recently been identified in foetal skeletal muscle. ${ }^{37}$ Over-expression of this isoform in this insulin-responsive tissue could more plausibly contribute to the growth restriction and lateral asymmetry phenotypes of SRS.

No sequence mutations were identified in the GRB10 coding region in 18 SRS cases screened. The possibility that epigenetic mutations of GRB10 are associated with SRS was not investigated. Recently, a nucleotide substitution resulting in a P95S change in GRB10 was identified in two unrelated Japanese SRS patients, but not in 100 normal controls. Functional studies will aid in determining the effect this substitution has on GRB10 activity, and whether it is a disease-causing mutation in these patients. ${ }^{38}$ The variability in the clinical presentation of SRS no doubt reflects the genetic heterogeneity of this disorder. However, in cases where the genetic aetiology involves a chromosome 7 disruption, more than one gene may contribute to different aspects of the syndrome. GRB10 is the first gene within the SRS candidate region of $7 \mathrm{p} 11.2$-p13 to be found to be imprinted, but sequence mutations appear to be exceedingly rare. Possible disease-causing mutations of GRB10 have only been identified in two of a total of 136 SRS patients screened, including the 18 cases in this report. This region of chromosome 7 is currently under investigation for other imprinted genes that may contribute to SRS.

\section{Acknowledgements}

We would like to thank Dr Susan Price for clinical assessment of the majority of SRS patients in the main SRS cohort, and Dr Emma Wakeling for providing normal adult fibroblast cDNAs for imprinting analysis. This work was supported by funding from the Dunhill Medical Trust (MP Hitchins) and Children Nationwide (D Monk).

\section{References}

1 Russell A: A syndrome of intrauterine dwarfism recognizable at birth with craniofacial dysostosis, disproportionate short arms and other anomalies. Proc R Soc Med 1954; 47: 1040-1044.

2 Silver HK, Kiyasu W, George J, Deamer WC: Syndrome of congenital hemihypertrophy, shortness of stature, and elevated urinary gonadotrophins. Pediatrics 1953; 12: 368-376.

3 Price SM, Stanhope R, Garrett C, Preece MA, Trembath RC: The spectrum of Silver-Russell syndrome: a clinical and molecular genetic study and new diagnostic criteria. J Med Genet 1999; 36: $837-842$.

4 Kotzot D, Schmitt S, Bernasconi F et al: Uniparental disomy 7 in Silver-Russell syndrome and primordial growth retardation. Hum Mol Genet 1995; 4: 583-587.

5 Preece MA, Price SM, Davies V et al: Maternal uniparental disomy 7 in Silver-Russell syndrome. J Med Genet 1997; 34: 6-9.

6 Eggermann T, Wollmann HA, Kuner R et al: Molecular studies in 37 Silver-Russell syndrome patients: frequency and etiology of uniparental disomy. Hum Genet 1997; 100: 415-419.

7 Preece MA, AbuAmero SN, Ali Z et al: An analysis of the distribution of hetero- and isodisomic regions of chromosome 7 in five mUPD7 Silver-Russell syndrome probands. J Med Genet 1999; 36: $457-460$.

8 Joyce CA, Sharp A, Walker JM, Bullman H, Temple IK: Duplication of 7p12.1-p13, including GRB10 and IGFBP1, in a mother and daughter with features of Silver-Russell syndrome. Hum Genet 1999; 105: $273-280$.

9 Monk D, Wakeling EL, Proud V et al: Duplication of 7p11.2-p13, including GRB10, in Silver-Russell syndrome. Am J Hum Genet 2000; 66: 36- 46 .

10 Eggermann K, Wollmann HA, Binder G, Kaiser P, Ranke MB, Eggermann T: Biparental expression of IGFBP1 and IGFBP3 renders their involvement in the etiology of Silver-Russell syndrome unlikely. Ann Genet 1999; 42: 117-121.

11 Wakeling EL, Hitchins MP, Abu-Amero SN, Stanier P, Moore GE, Preece MA: Biallelic expression of IGFBP1 and IGFBP3, two candidate genes for the Silver-Russell syndrome. I Med Genet 2000; 37: 65-67

12 Eggermann K, Wollmann HA, Tomiuk J, Ranke MB, Kaiser P, Eggermann T: Screening for mutations in the promoter and the coding region of the IGFBP1 and IGFBP3 genes in Silver-Russell syndrome patients. Hum Hered 1999; 49: 123-128.

13 Miyoshi N, Kuroiwa Y, Kohda T et al: Identification of the Meg1/ Grb10 imprinted gene on mouse proximal chromosome 11, a candidate for the Silver-Russell syndrome. Proc Natl Acad Sci USA 1998; 95: 1102-1107. 
14 Liu F, Roth RA: Grb IR: a SH2-domain-containing protein that binds to the insulin receptor and inhibits its function. Proc Natl Acad Sci USA 1995; 92: 10287 - 10291.

15 O'Neill TJ, Rose DW, Pillay TS, Hotta K, Olefsky JM, Gustafson TA: Interaction of a GRB-IR splice variant (a human GRB10 homologue) with the insulin-like growth factor I receptors. $J$ Biol Chem 1996; 271: 22506-22513.

16 Frantz JD, Giorgetti-Peraldi S, Ottinger EA, Schoelson SE: Human GRB-IR/GRB10. J Biol Chem 1997; 272: 2659-2667.

17 Dong LQ, Du H, Porter SG et al: Cloning, chromosome localization, expression, and characterization of an Src homology 2 and pleckstrin homology domain-containing insulin receptor binding protein hGrb10 $\gamma$. J Biol Chem 1997; 272: 29104-29112.

18 He W, Rose DW, Olefsky JM, Gustafson TA: Grb10 interacts differentially with the insulin receptor, insulin-like growth factor I receptor, and epidermal growth factor receptor via the Grb10 Src homology 2 (SH2) domain and a second novel domain located between the pleckstrin homology and $\mathrm{SH} 2$ domains. Biol Chem 1998; 273: 6860-6867.

19 Morrione A, Valentinis B, Resnicoff M, Xu S, Baserga R: The role of $\mathrm{mGrb} 10 \alpha$ in insulin-like growth factor I-mediated growth. J Biol Chem 1997; 272: 26382-26387.

20 Moutoussamy S, Renaudie F, Lago F, Kelly PA, Finidori J: Grb10 identified as a potential regulator of growth hormone $(\mathrm{GH})$ signaling by cloning of GH receptor target proteins. J Biol Chem 1998; 273: $15906-15912$.

21 Jerome CA, Scherer SW, Tsui L-C, Gietz RD, Triggs-Raine B: Assignment of growth factor receptor-bound protein 10 (GRB10) to human chromosome 7p11.2-p12. Genomics 1997; 40: $215-216$.

22 Cattanach BM, Kirk M: Differential activity of maternally and paternally derived chromosome regions in mice. Nature 1985; 315: $496-498$

23 Kunkel LM, Smith KD, Boyer SH et al: Analysis of human Ychromosome-specific reiterated DNA in chromosome variants. Proc Natl Acad Sci USA 1977; 74: 1245-1249.

24 Chirgwin JM, Przybyla AE, MacDonald RJ, Rutter WJ: Isolation of biologically active ribonucleic acid from sources enriched in ribonuclease. Biochemistry 1979; 18: 5294-5299.

25 Angrist M, Bolk S, Bentley K, Nallasamy S, Halushka MK, Chakravarti A: Genomic structure of the gene for the SH2 and pleckstrin homology domain-containing protein GRB10 and evaluation of its role in Hirschsprung disease. Oncogene 1998; 17: $3065-3070$.
$26 \mathrm{Vu} \mathrm{TH}$, Hoffmann AR: Promoter-specific imprinting of the human insulin-like growth factor II gene. Nature 1994; 371 : $714-717$.

27 Riesewijk AM, Hu L, Schulz U et al: Monoallelic expression of human PEG1/MEST is paralleled by parent-specific methylation in fetuses. Genomics 1997; 42: 236-244.

28 Kosaki K, Kosaki R, Craigen WJ, Matsuo N: Isoform-specific imprinting of the human PEG1/MEST gene. Am J Hum Genet 2000; 66: 309-312.

29 Wutz A, Smrzka OW, Schweifer N, Schellanders K, Wagne EF, Barlow DP: Imprinted expression of the $I g f 2 r$ gene depends on an intronic CpG island. Nature 1997; 389: 745 - 749.

30 Rougelle C, Cardoso C, Fontes M, Colleaux L, Lalande M: An imprinted antisense RNA overlaps $U B E 3 A$ and a second maternally expressed transcript. Nat Genet 1998; 19: 15-16.

31 Wroe SF, Kelsey G, Skinner JA et al: An imprinted transcript, antisense to Nesp, adds complexity to the cluster of imprinted genes at the mouse Gnas locus. Proc Natl Acad Sci USA 2000; 97 $3342-3346$

32 Rougelle $C$, Glatt $H$, Lalande $M$ : The Angelman syndrome candidate gene, $U B E 3 A / E 6-A P$, is imprinted in brain. Nat Genet 1997; 17: $14-15$.

$33 \mathrm{Vu} \mathrm{TH}$, Hoffmann AR: Imprinting of the Angelman syndrome gene, UBE3A, is restricted to brain. Nat Genet 1997; 17: 12-13.

34 Albrecht U, Sutcliffe JS, Cattanach BM et al: Imprinted expression of the murine Angelman syndrome gene, beE3a, in hippocampal and Purkinje neurons. Nat Genet 1997; 17: 75 - 78

35 Matsuoka S, Thompson JS, Edwards MC et al. Imprinting of the gene encoding a human cyclin-dependent kinase inhibitor, p57KIP2, on chromosome 11p15. Proc Natl Acad Sci USA 1996; 93: $3026-3030$

36 Kalscheuer VM, Mariman EC, Schepens MT, Rehder H, Ropers H$\mathrm{H}$ : The insulin-like growth factor type-2 receptor gene is imprinted in the mouse but not in humans. Nat Genet 1993; 5: $74-78$.

37 Blagitko N, Mergenthaler S, Schulz U et al: Human GRB10 is imprinted and expressed from the paternal and maternal allele in a highly tissue- and isoform-specific fashion. Hum Mol Genet 2000; 9: $1587-1595$.

38 Yoshihashi H, Maeyama K, Kosaki R et al: Imprinting of human GRB10 and its mutations in two patients with Russell-Silver syndrome. Am J Hum Genet 2000; 67: 476-482. 